\title{
Factores determinantes de cumplimiento terapéutico en adultos mayores hipertensos
}

\author{
Sara Mendoza Pla, Mónica Muñoz $P^{1 b}$, José $M$ Merino $E^{2 c}$, \\ O mar A Barriga2d. \\ Determinant factors of therapeutic \\ compliance in elderly hypertensive \\ patients
}

Background: In Chile, less than $30 \%$ of treated hypertensive patients achieve normal blood pressure values. Lack of compliance with treatment may influence the lack of success of treatments. Aim: To identify determinant treatment compliance factors, according to the Health Belief Model, in elderly hypertensive patients. Material and methods: A stratified and randomized sample with 245 elderly hypertensive patients, participating in the Cardiovascular Program of the San Pedro de la Paz Health Center in Chile, was studied. Modified Miller's Health Behavior Scale to measure therapeutic compliance and Health Belief Questionnaire to measure independent factors influencing compliance, were applied. Data analysis was done with Student's, test ANOVA and linear regression. Results: Eight of 23 factors were predictors of compliance: gender, occupational activity, smoking, modification of health behaviors, difficulties to take medications, perception of treatment effectiveness, knowledge and perception about hypertension. Conclusions: Knowledge about factors that influence therapeutic compliance allows to modify health care programs and improve their effectiveness (Rev Méd Chile 2006; 134: 65-71).

(Key w ords: Aged; Hypertension; Patient acceptance of health care; Patient compliance)

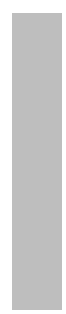

Recibido el 14 de diciembre, 2004. Aceptado el 26 de julio, 2005.

Proyecto de Investigación con financiamiento de la Dirección de Investigación de la Universidad de Concepción (DIUC 200.082.024-1.0).

${ }^{1}$ Departamento de Enfermería, Facultad de Medicina, Universidad de Concepción. ${ }^{2}$ Departamento de Sociología, Facultad de Ciencias Sociales, Universidad de Concepción.

aEnfermera. Candidata a Doctora en Enfermería.

bEnfermera. Magister en Enfermería.

'Sociólogo, PhD.

dLicenciado en RR.II, PhD. en Sociología

Correspondencia a: Sara Mendoza P. Departamento de Enfermería, Facultad de Medicina, Universidad de Concepción, Concepción. Fono: (56-41) 204948. Fax: (5641) 228353. E mail: smendoza@udec.cl. 
$\mathrm{E}^{1}$ proceso de transición que experimenta nuestro país a nivel demográfico y epidemiológico, ha obligado al Ministerio de Salud a abordar de manera estratégica las necesidades de salud de los adultos mayores ${ }^{1}$. La creciente longevidad está asociada a bajos niveles de calidad de vida ${ }^{2}$, fundamentalmente por la mayor incidencia de enfermedades crónicas $^{3}$, entre las que está la hipertensión arterial (HTA), que es un proceso que exige adaptar una terapia sistemática, basada en cambios drásticos de estilos de vida.

Si los adultos mayores hipertensos cumplen la terapia e, idealmente, eliminan factores de riesgo (tabaquismo, sedentarismo u obesidad), pueden reducir la tasa de complicaciones y prolongar la sobrevida 4 .

El incumplimiento terapéutico, contrariamente, aumenta la gravedad y acorta la vida de muchos pacientes. Aunque la literatura científica respecto al cumplimiento terapéutico se ha venido incrementando, son escasos los estudios focalizados en entender cómo se manifiesta esta conducta en adultos mayores con padecimientos cardiovasculares $^{5}$.

Si se considera que el adulto mayor hipertenso ha acumulado creencias, costumbres y hábitos durante, al menos, 60 años, cuyo resultado es la enfermedad, surgió la necesidad de estudiar qué es lo que determina la adopción de una conducta terapéutica cumplidora cuando se ha diagnosticado HTA.

Investigar esta conducta es importante porque el control de la enfermedad a escala mundial oscila sólo entre 10 y $25 \%$ y entre los factores principales de esta situación, está el incumplimiento fármaco-terapéutico, aspecto crítico, si pensamos en la cadena del medicamento: elaboración-prescripción/indicación-dispensación-consumo, porque no ingerir el fármaco, significa anular todo el esfuerzo anterior ${ }^{6}$.

En Chile, existe un bajo porcentaje de éxito (inferior a 30\%) en obtener cifras iguales 0 inferiores a 140/90 mmHg (límites actuales de normalidad de la presión arterial) en pacientes hipertensos $^{7}$, por lo que se puede inferir la magnitud del problema, cuando se sabe que las complicaciones aumentan con la edad y el envejecimiento puede contribuir a elevar la mortalidad ${ }^{8}$.

De acuerdo con las actuales recomendaciones de la $\mathrm{OMS}^{9}$, el cumplimiento terapéutico o adhe- rencia debe entenderse como una conducta en la que el paciente participa racionalmente en todas las recomendaciones dadas por el grupo de profesionales para tratar su enfermedad.

Un modelo teórico que permite entender esta conducta es el "Modelo de creencias en salud" (MCS), porque explica el proceso de toma de decisiones respecto a salud e identifica factores (motivacionales, subjetivos, elementos habilitantes o modificantes de la conducta) que actuarían como determinantes de una mayor o menor probabilidad de cumplir las prescripciones para tratar la enfermedad ${ }^{10}$.

Este estudio, basado en el MCS, se propuso determinar el modelo óptimo de factores predictores del cumplimiento terapéutico en adultos mayores hipertensos, objetivo en que se utilizaron 23 variables para establecer las hipótesis de trabajo (Tabla 1).

\section{MATERIAL Y MÉTODOS}

Se diseñó un estudio observacional, cuya unidad de análisis fue el paciente hipertenso adulto mayor (65 años o más) ingresado al Programa Cardiovascular del Centro de Salud San Pedro, Comuna San Pedro de la Paz, VIII Región, Chile. Del universo $(\mathrm{N}=725)$ se obtuvo una muestra estratificada al azar del 33,8\% ( $\mathrm{n}=245)$. El criterio de inclusión fue: pacientes con mínimo dos años de ingreso, para resguardar que hubieran tenido tiempo suficiente en relacionarse con el programa y los profesionales. Se excluyeron aquellos pacientes con domicilios falsos o fuera de los límites comunales, con manejo ambulatorio sólo a nivel secundario y con alguna condición física o síquica que impidiera su llegada periódica al centro de salud (Tabla 1).

Previo consentimiento informado, se aplicaron dos instrumentos en el hogar de cada adulto mayor hipertenso:

a) Escala de Conductas en Salud de Miller ${ }^{11}$, para medir la variable dependiente "cumplimiento terapéutico". Esta escala, validada en Chile en pacientes infartados $^{12}$ e hipertensos ${ }^{13}$, informa sobre cinco aspectos considerados en las indicaciones de la hipertensión (dieta, consumo de tabaco, actividad física, medicamentos y estrés) propuestos en 4 enunciados que indagan el cumplimiento en la casa, 
Tabla 1. M odelo de creencias en salud y derivación de variables que fueron consideradas en cada hipótesis de trabajo como factores predictores de cumplimiento terapéutico en adultos mayores hipertensos

\begin{tabular}{|c|c|c|}
\hline & Modelo de creencias en salud & Factores \\
\hline \multirow{3}{*}{$\begin{array}{l}\text { Disposición a } \\
\text { adoptar la } \\
\text { conducta de rol } \\
\text { de enfermo }\end{array}$} & Motivaciones & $\begin{array}{l}\text { Importancia concedida a la salud en general } \\
\text { Modificación de hábitos de salud } \\
\text { Cantidad de controles }\end{array}$ \\
\hline & $\begin{array}{l}\text { Valor dado a la reducción de } \\
\text { la amenaza de la enfermedad }\end{array}$ & $\begin{array}{l}\text { Percepciones de la HTA } \\
\text { Percepción de la severidad de la HTA } \\
\text { Percepción de resuceptibilidad a crisis hipertensiva }\end{array}$ \\
\hline & $\begin{array}{l}\text { Probabilidad que la conducta } \\
\text { reducirá la amenaza }\end{array}$ & Percepción de la eficacia del régimen de tratamiento \\
\hline \multirow{7}{*}{$\begin{array}{l}\text { Factores } \\
\text { modificantes y } \\
\text { habilitantes }\end{array}$} & Sociodemográficos y de salud & $\begin{array}{l}\text { Sexo, edad, estado marital, religión, nivel educacional } \\
\text { actividad laboral y hábito de fumar }\end{array}$ \\
\hline & Estructurales & Costo financiero \\
\hline & & $\begin{array}{l}\text { Adaptacion a la duracion del tratamiento } \\
\text { Compleiidad del tratamiento medicamentoso }\end{array}$ \\
\hline & Actitudes & Dificultades en la ingesta del medicamento \\
\hline & Interacción & $\begin{array}{l}\text { Satisfacción con aspectos contextuales del control } \\
\text { Satisfacción con la calidad de la atención }\end{array}$ \\
\hline & Habilitación & $\begin{array}{l}\text { Satisfacción con interacción médico-paciente } \\
\text { Satisfacción con interacción enfermera/o-paciente }\end{array}$ \\
\hline & & Conocimiento de la enfermedad hipertensiva \\
\hline
\end{tabular}

trabajo, actividades recreativas y sociales. El puntaje fluctúa entre 12 y 60 puntos. Aunque se utiliza en dos versiones: un auto-informe del paciente y un informe de un familiar, a objeto de validar la respuesta del primero, se constató que una gran mayoría de adultos mayores, sobre todo mujeres, viven solos o solas, por lo que no se podían obtener las respuestas del familiar. En estudios anteriores se encontró una alta correlación entre las respuestas del paciente y las del familiar ${ }^{15}$, por lo tanto, no fue considerado problema prescindir del instrumento proveniente del familiar. La confiabilidad de la escala de cumplimiento terapéutico, medida con Alpha de Cronbach arrojó los siguientes valores: subescala dieta $=0,89$; subescala actividad $=0,95$; subescala medicamento $=0,92$ y subescala estrés $=0,92$.

b) Cuestionario de creencias en salud para pacientes hipertensos ${ }^{13}$, que recopiló datos de las variables independientes.
Para comprobar las hipótesis, considerando al cumplimiento terapéutico como una variable dependiente continua, que tuvo un comportamiento normal, se aplicaron modelos lineales generales.

Para el procesamiento y análisis de datos se usó SPSS 11.5.

\section{RESULTADOS}

Comportamiento de la muestra. Sólo $21,6 \%$ de la muestra alcanzó el puntaje máximo en todas las subescalas, lo que por complemento indica una alta prevalencia de incumplimiento terapéutico $(78,4 \%)$.

Las variables sociodemográficas, evidenciaron el perfil típico del grupo adulto mayor en Chile actual $^{14}$, compuesto por una mayoría femenina, que sustenta la viudez de la tercera edad (de 37\% de viudos, $29 \%$ eran mujeres) y una educación 
que no supera el nivel básico. Entonces estamos frente a un grupo desprovisto del contexto familiar y del nivel educacional adecuado para manejar la HTA, pues se sabe que el apoyo familiar se vincula con el apego al tratamiento de cualquier enfermedad $^{15}$. Si sumamos las disfunciones propias del envejecimiento, podríamos inferir que el problema del incumplimiento, sobre todo en la mujer adulta mayor, se instala por soledad, desconocimiento y deterioro.

Llama la atención que casi un tercio de los adultos mayores no sabía que su enfermedad es de por vida. Esto implica la necesidad de reforzar la educación acerca de lo que significa una enfermedad crónica, pues se sabe que el cumplimiento terapéutico disminuye con los años y un enfermo que aún no está adaptado a esta condición, puede tener menos posibilidades de seguir las indicaciones en un largo plazo ${ }^{13}$.

Los hábitos que mayoritariamente se habían modificado en el grupo eran los referidos a alimentación y consumo de medicamentos, (82\% y $89 \%$, respectivamente) mientras que los referi- dos al manejo de conflictos personales o recreación sólo se habían modificado en 50\% y 40\%, respectivamente. Esto revela que un gran porcentaje de hipertensos no considera los aspectos sociales o psicológicos que ayudan a manejar la enfermedad, estando muy arraigada la idea que los tratamientos o los actos médicos culminan con la prescripción farmacológica y se deposita en ese aspecto casi toda la solución a la enfermedad. Esto pudiera tener explicación en el manejo que el mismo equipo de salud implementa para los pacientes hipertensos, pues en este estudio llamó la atención que todos los casos tenían indicados medicamentos antihipertensivos desde la fecha de ingreso al programa, sin resguardar los tres a seis meses de manejo no-farmacológico inicial, necesario y recomendado para esta edad ${ }^{16}$, situación que pudiera estar favoreciendo que el paciente perciba que sólo la prescripción farmacológica es importante en su tratamiento.

Coincidiendo con lo encontrado por enfermeras norteamericanas ${ }^{5}$, sólo 45,3\% manifestó estar satisfecho con la sala de espera, box de atención, baños y

Tabla 2. Pruebas T de Student para variables categóricas binarias que resultaron predictoras de cumplimiento terapéutico

\begin{tabular}{|c|c|c|c|c|c|c|c|c|c|}
\hline $\begin{array}{l}\text { Variables } \\
\text { independientes } \\
\text { (predictores) }\end{array}$ & Categorías & $\mathrm{N}$ & Media & DS & $\begin{array}{l}\text { Err. tip. } \\
\text { Media }\end{array}$ & $\begin{array}{c}\text { Sig. } \\
\text { Levene }^{\circ}\end{array}$ & $\mathrm{t}$ & gl & $\mathrm{p}^{*}$ \\
\hline \multirow[t]{2}{*}{ Sexo } & Femenino & 170 & 30,6 & 5,38 & 0,41 & \multirow{2}{*}{0,278} & \multirow{2}{*}{$-4,513$} & \multirow{2}{*}{243} & \multirow{2}{*}{0,000} \\
\hline & Masculino & 75 & 33,9 & 5,30 & 0,61 & & & & \\
\hline \multirow[t]{2}{*}{ Sit. Laboral } & Trabajo remunerado & 8 & 44,6 & 4,84 & 1,71 & \multirow[b]{2}{*}{0,942} & \multirow[b]{2}{*}{7,399} & \multirow[b]{2}{*}{243} & \multirow[b]{2}{*}{0,000} \\
\hline & No trabaja & 237 & 31,2 & 5,04 & 0,33 & & & & \\
\hline \multirow[t]{2}{*}{$\begin{array}{l}\text { Hábito de } \\
\text { fumar }\end{array}$} & Fuma & 27 & 35,6 & 5,18 & 1,00 & \multirow[t]{2}{*}{0,600} & \multirow[t]{2}{*}{4,009} & \multirow[t]{2}{*}{243} & \multirow[t]{2}{*}{0,000} \\
\hline & No fuma & 218 & 31,2 & 5,43 & 0,37 & & & & \\
\hline \multirow[t]{2}{*}{$\begin{array}{l}\text { Percepción de } \\
\text { la HTA }\end{array}$} & Siente la HTA & 99 & 30,4 & 5,75 & 0,58 & \multirow[t]{2}{*}{0,231} & \multirow[t]{2}{*}{$-3,031$} & \multirow[t]{2}{*}{243} & \multirow[t]{2}{*}{0,003} \\
\hline & No siente la HTA & 146 & 32,5 & 5,28 & 0,44 & & & & \\
\hline $\begin{array}{l}\text { Dificultades en } \\
\text { ingesta de } \\
\text { medicamentos }\end{array}$ & $\mathrm{C} /$ dificultades & 15 & 27,9 & 5,28 & 1,36 & 0,766 & $-2,712$ & 243 & 0,007 \\
\hline
\end{tabular}

- varianzas iguales en todos los factores.

* alpha $\leq 0,05$ indica aprobación hipótesis conceptual planteada para cada una de las variables que muestra la tabla. 
tiempo de espera, siendo estos últimos, los aspectos que provocaban la mayor frecuencia de insatisfacción (33,1\% y 31,8\%, respectivamente). Lo anterior es importante, puesto que para el paciente hipertenso que concurre a su control y debe permanecer en una sala de espera, la necesidad de un baño, aumenta con el paso de las horas y por evitar ir al baño en el centro de salud, el paciente no ingerirá el medicamento, pudiendo ser calificado falsamente como incumplidor cuando se le controle la presión arterial y una valoración errónea puede dar pie a establecer cambios errados en el tratamiento (aumentar dosis, eliminar tratamientos eficaces) y almacenamiento innecesario de medicamentos no usados por el paciente.

Predictores. De las 23 variables del estudio, ocho tuvieron un comportamiento predictor.

La Tabla 2 muestra cinco factores del MCS que resultaron predictores de cumplimiento terapéutico para el grupo de estudio: el sexo, la situación laboral y el hábito de fumar, representan los predictores sociodemográficos y de salud del modelo; las dificultades en la ingesta de medicamentos representa a las variables estructurales y la percepción de la HTA representa un factor cognitivo que reduciría la amenaza de la enfermedad en los adultos mayores.

La Tabla 3 muestra tres variables que se suman a las que resultaron predictoras para el cumplimiento terapéutico: modificación de hábitos de salud, conocimiento de la enfermedad y percepción de eficacia del régimen de tratamiento propuesto; la primera, representa la motivación a adoptar la conducta cumplidora, la segunda contribuiría como variable habilitadora del cumplimiento y la tercera representa características que favorecen el cumplimiento.

La organización de los resultados encontrados en la perspectiva teórico-conceptual del MCS, indica que en nuestro estudio los predictores determinantes del cumplimiento terapéutico en adultos mayores hipertensos se expresa como se demuestra en la Figura 1.

Tabla 3. Prueba de regresión lineal para variables continuas que resultaron predictoras de cumplimiento terapéutico

\begin{tabular}{|c|c|c|c|c|c|c|c|c|c|c|}
\hline $\begin{array}{l}\text { Variable } \\
\text { independiente } \\
\text { (predictores) }\end{array}$ & $\mathrm{R}$ & $\mathrm{R}^{2}$ & $\begin{array}{c}\mathrm{R}^{2} \\
\text { Ajust }\end{array}$ & $\begin{array}{l}\text { Err } \\
\text { tip. } \\
\text { estim }\end{array}$ & Modelo & Beta & B & $\begin{array}{l}\text { Error } \\
\text { típ. B }\end{array}$ & $\mathrm{t}$ & $\mathrm{p}^{*}$ \\
\hline $\begin{array}{l}\text { Modificación } \\
\text { hábitos en } \\
\text { salud }\end{array}$ & 0,169 & 0,029 & 0,025 & 5,50 & $\begin{array}{l}\text { Intercepto } \\
\text { modificación } \\
\text { hábitos }\end{array}$ & $\overline{-}$ & $\begin{array}{c}29,530 \\
0,631\end{array}$ & $\begin{array}{l}0,870 \\
0,236\end{array}$ & $\begin{array}{l}33,94 \\
2,678\end{array}$ & $\begin{array}{l}0,000 \\
0,008\end{array}$ \\
\hline $\begin{array}{l}\text { Percepción } \\
\text { severidad } \\
\text { HTA }\end{array}$ & 0,118 & 0,14 & 0,010 & 5,54 & $\begin{array}{l}\text { Intercepto } \\
\text { percepción } \\
\text { severidad }\end{array}$ & - & $\begin{array}{l}33,909 \\
-2,210\end{array}$ & $\begin{array}{l}1,260 \\
0,113\end{array}$ & $\begin{array}{l}26,92 \\
-1,86\end{array}$ & $\begin{array}{l}0,000 \\
0,064^{*}\end{array}$ \\
\hline $\begin{array}{l}\text { Conocimiento } \\
\text { de la HTA }\end{array}$ & 0,125 & 0,016 & 0,012 & 5,53 & $\begin{array}{l}\text { Intercepto } \\
\text { conocimiento }\end{array}$ & $\begin{array}{c}- \\
0,125\end{array}$ & $\begin{array}{c}29,788 \\
0,916\end{array}$ & $\begin{array}{l}1,018 \\
0,467\end{array}$ & $\begin{array}{c}29,26 \\
1,96\end{array}$ & $\begin{array}{l}0,000 \\
0,051\end{array}$ \\
\hline $\begin{array}{l}\text { Percepción de } \\
\text { eficacia del } \\
\text { tratamiento }\end{array}$ & 0,156 & 0,024 & 0,020 & 5,51 & $\begin{array}{l}\text { Intercepto } \\
\text { percepción } \\
\text { eficacia }\end{array}$ & $\overline{-}$ & $\begin{array}{c}28,278 \\
0,412\end{array}$ & $\begin{array}{l}1,421 \\
0,167\end{array}$ & $\begin{array}{l}19,9 \\
2,46\end{array}$ & $\begin{array}{l}0,000 \\
0,015\end{array}$ \\
\hline
\end{tabular}

*valor considerado marginalmente significativo. 

Disposición a adoptar
Factores modificantes
Conducta de rol
conducta de rol de
y habilitantes
de enfermo
enfermo hipertenso
hipertenso

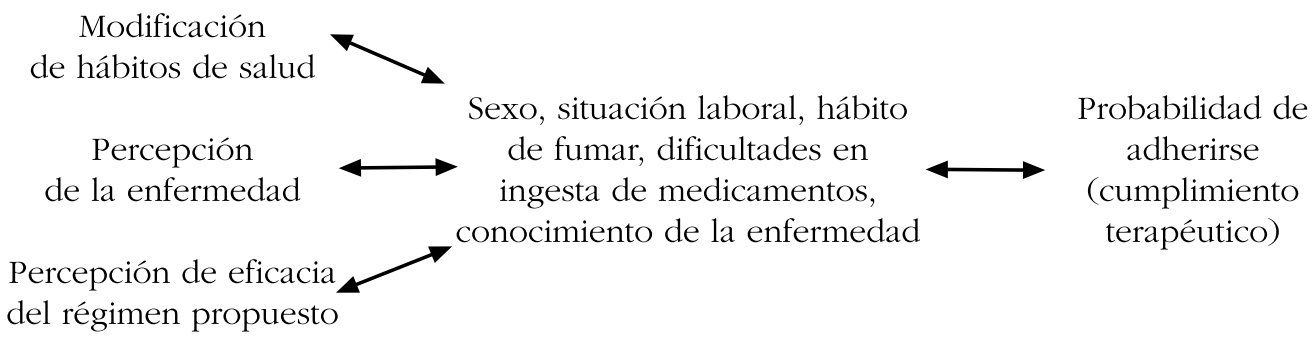

Figura 1. Aplicación del "modelo de creencias en salud" en su versión extendida a la conducta de rol de enfermo, en adultos mayores hipertensos del Programa Cardiovascular del Centro de Salud San Pedro, Comuna de San Pedro de la Paz, VIII Región, Chile 2004.

\section{DisCUSIÓN}

La alta prevalencia de incumplimiento terapéutico encontrada en el grupo de estudio, puede ser explicada por la capacidad que tiene la "Escala en conductas en salud" de Miller en indagar rigurosamente el comportamiento terapéutico que adopta el paciente en todas las actividades de su vida diaria y coincide con el bajo porcentaje de éxito $(<30 \%)$ que se tiene en el país para obtener que la población hipertensa alcance cifras tensionales iguales o inferiores a $140 / 90 \mathrm{mmHg}^{7}$.

El género resultó predictor $(t=4,513 ; \mathrm{p}=0,000) \mathrm{y}$ coincide con estudios en mujeres norteamericanas ${ }^{17}$. Los hombres alcanzaron mayores puntajes de cumplimiento respecto a las mujeres y son, además, el grupo que aún trabaja en esta edad, actividad que también resultó ser predictora $(\mathrm{t}=7,399 ; \mathrm{p}=0,000)$. Son las mujeres las que tienen menos probabilidades de ser cumplidoras al llegar a la tercera edad, situación que podría explicarse por los roles diferenciados de género que se adoptan en el cuidado de la salud de la familia o en la pareja. Si un hombre es hipertenso, el cuidado de la mujer aumenta sus probabilidades de cumplir un tratamiento, pero si es la mujer la hipertensa, cquién la apoya en el cuidado de su salud?

Otro aspecto importante de considerar es la predicción que se obtuvo en la variable que medía la dificultad en la ingesta $(t=2,712 ; p=0,007)$ v/s la que medía la complejidad en base al número de medicamentos $(\mathrm{F}=1,59 ; \mathrm{p}=0,21)$, lo que indica que para este grupo, no es la cantidad de medicamentos lo que incide en el cumplimiento, sino más bien la complicación que la ingesta le ocasiona. Estudios similares en adultos mayores hipertensos norteamericanos han revelado que el olvido y los efectos desagradables que les producen los antihipertensivos, son las dificultades que impiden cumplir con la ingesta de medicamentos $^{5}$. Estas dificultades, aunque no se indagaron en profundidad, pueden originarse al momento de la ingesta (dificultades para tragar, confusión de dosis, etc.) o después, por efectos no deseados, como el deseo de orinar más frecuentemente, situaciones que provocan a la larga la decisión de evitar la ingesta y ahorrarse las molestias de una enfermedad que ni siquiera perciben. Sobre todo en el anciano, la ingesta de medicamentos y la ocurrencia de efectos secundarios, deben ser permanentemente valorados por el equipo de salud, para ir adecuando el tratamiento integral a las necesidades y actividades diarias del paciente.

Queda residualmente comprobado que conocer la HTA $(t=1,96 ; p=0,051)$ determina el cumplimiento terapéutico, conocimiento que en el adulto mayor debe ser permanentemente reforzado, considerando las capacidades cognitivas, el tiempo transcurrido desde el diagnóstico y las percepciones de la enfermedad ${ }^{17,18}$. A través de mensajes claros, específicos y bien estructurados se puede traspasar el manejo de la enfermedad hacia el paciente y lograr mayor adherencia y la clave para ello está en desarro- 
llar relaciones más abiertas y cooperativas con el paciente ${ }^{19}$. La comprensión de las dificultades del paciente por parte del personal que entrega el cuidado, es esencial para guiar los contenidos educativos y lograr un adecuado cumplimiento terapéutico ${ }^{20}$.

La percepción de gravedad no resulta menos relevante, aunque estadísticamente sólo resultó marginalmente significativa ( $t=1,86 ; p=0,064)$ : los valores alcanzados indican que el adulto mayor hipertenso capta lo grave que puede resultar su

\section{REFERENCIAS}

1. MINSAL Cuidado de la Salud del Adulto Mayor en el Nivel Primario. Programa de Salud del Adulto. Chile: Ministerio de Salud; 1996.

2. Schwartzmann L Calidad de vida relacionada con la salud: aspectos conceptuales. Ciencia y Enfermería 2003; 9: 9-21.

3. Bazo ME. La ancianidad del futuro. Madrid: SG Editores SA; 1992.

4. Román O. Riesgo cardiovascular de la hipertensión arterial. Hipertensión 1991; 7.

5. Evangelista LS, Doering LV, Dracup K, Westlake C, Hamilton M, Fonarow G. Compliance behavior of elderly patients with advances heart failure. Journal of Cardiovascular Nursing 2003; 18: 197-206.

6. BASTERRA MG. El cumplimiento terapéutico. Pharm Care Esp 1999; 1: 97-106.

7. Román OA, Valenzuela MAC, Badi山a MS, Báez DC, HERRERA EV. Optimización de la reducción de la presión arterial en hipertensos esenciales. Rev Méd Chile 2002; 130: 519-26.

8. Román OA, Badila MS, Valenzuela AC, Cumsille FG, Rodríguez SN. Perfil del hipertenso adulto mayor tratado. Rev Méd Chile 2002; 130: 616-22.

9. SABATÉ E (eDITOR). Adherence to long-term therapies: evidence for action. [documento en Internet]. Geneva: The WHO Adherente to Long-term Therapies Project 2003. World Health Organization Health Care for Chronic Conditions (CCH); 2003 WHO/MNC/03.01. Disponible en URL <http:// www.who.int/chronic conditions/adherencereport/en/ >. [Consultado el 7 de agosto 2004].

10. BeCKer MH. The Health Belief Model and Personal Health Behavior. USA: CBS; 1974. enfermedad si no cumple las indicaciones prescritas. Cuando el paciente percibe lo grave de la enfermedad o se siente enfermo, tiene mayor probabilidad de cumplir la terapia ${ }^{17}$, probabilidad que aumentará, si percibe que el tratamiento que le ha sido indicado será beneficioso ${ }^{21}$.

Se recomienda seguir indagando esta conducta permanentemente, sobre todo en la población adulta mayor femenina, que aumenta, envejece y enfrenta esta enfermedad crónica en las peores condiciones de vida: sola y pobre.

11. Miler P, Wikoff RL, Mcmahon M, GarRet MJ, Ringel $\mathrm{K}$. Indicators of medical regimen adherence for myocardial infarction patients. Nursing Research 1985; 34: 268-72.

12. JARA P. Efectos de una intervención de enfermenía en la adherencia al tratamiento y control del paciente con IAM. Horizonte de Enferm 1996; 7: 19-25.

13. Mendoza PS, Figueroa FM. Creencias en salud y adherencia de pacientes hipertensos controlados en centros de salud de la comuna de Concepción. Ciencia y Enferm 1998; 4: 63-76.

14. Muñoz PM, Mendoza PS, Salazar MA. Perfil biosociodemográfico y dependencia funcional del adulto mayor. Gerokomos 2004; 15: 18-27.

15. Marín-Reyes F, Rodríguez-Morán M. Apoyo familiar en el apego al tratamiento de la hipertensión arterial esencial. Salud Pública Méx 2001; 43: 336-9.

16. Abelí́n MA, Leal H, Hernández FM, García-Galbis JAM, MaRTínez AP. Hipertensión en el anciano. Hipertensión 2001; 18: 78-85.

17. GRANT M. Women's health beliefs regarding hypertension. West Indian Med J 1993; 42: 158-60.

18. MAELAND JG. Changing patient's health behavior consultation and physician patient relationship. Tidsskr Nor Laegeforen 1993; 113: 47-50.

19. Donovan JL, Blake DR. Patient non-compliance: deviance or reasoned decision-making? Soc Sci Med 1992; 34: 507-11.

20. Clarence M. Ensuring compliance with drug regimens. Nurs-Stand 1992; 7: 24-6.

21. Silva SM, Valsechi EAA, Torra Silva RLD, Waidman MAP. Comportamiento preventivo de Servidores da U.E.M. Hipertensos e a Aderencia ao Programa de Assistencia ao Hipertenso do Ambulatorio. Ciencia y Enferm 1995; 1: 33-42. 